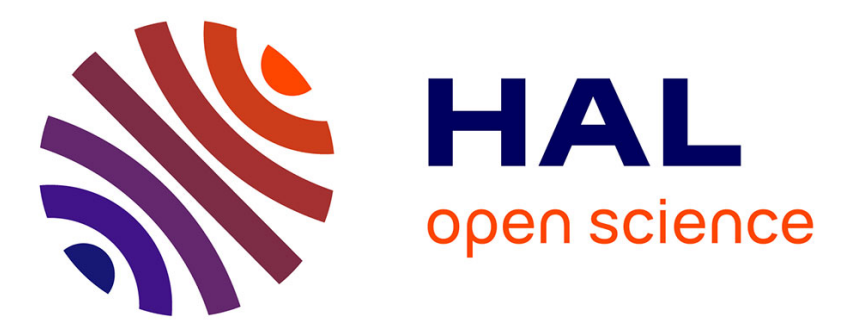

\title{
Robustness measure for fuzzy maintenance activities schedule.
}

François Marmier, Christophe Varnier, Noureddine Zerhouni

\section{To cite this version:}

François Marmier, Christophe Varnier, Noureddine Zerhouni. Robustness measure for fuzzy maintenance activities schedule.. 4th IFAC Conference on Management and Control of Production and Logistics, MCPL'2007., Sep 2007, Sibiu, Romania. pp.85-90. hal-00181107

\section{HAL Id: hal-00181107 https://hal.science/hal-00181107}

Submitted on 23 Oct 2007

HAL is a multi-disciplinary open access archive for the deposit and dissemination of scientific research documents, whether they are published or not. The documents may come from teaching and research institutions in France or abroad, or from public or private research centers.
L'archive ouverte pluridisciplinaire HAL, est destinée au dépôt et à la diffusion de documents scientifiques de niveau recherche, publiés ou non, émanant des établissements d'enseignement et de recherche français ou étrangers, des laboratoires publics ou privés. 


\title{
ROBUSTNESS MEASURE FOR FUZZY MAINTENANCE ACTIVITIES SCHEDULE
}

\author{
François MARMIER *, Christophe VARNIER *, \\ Noureddine ZERHOUNI* \\ * Laboratoire d'Automatique de Besançon (LAB), UMR \\ CNRS 6596 - ENSMM/UFC, 24 rue Alain Savary, 25000 \\ Besançon, FRANCE \\ \{Fmarmier, Cvarnier, Nzerhouni\}@ens2m.fr
}

\begin{abstract}
Skills management in industry is one of the most important factors in order to obtain good performance with production means. Especially in the field of maintenance services where the different practical knowledge or skills are their working tools. We address, in this paper, both the assignment and scheduling problems that can be found in a maintenance service. Each task that has to be performed is characterized by the level of skill required. The problem lies with making the decision of which time is the right time for the assignment and scheduling of the correct resource to do the task. For human resources, all skill levels are different, they are considered as unrelated parallel machines. Our aim is to assign new tasks to the adequate resources by giving to the maintenance expert a good and robust possibility.
\end{abstract}

Keywords: Human Resources, Maintenance, Scheduling, Skill, Uncertainties

\section{INTRODUCTION}

To stay competitive, companies must decrease their costs as much as possible and optimize their production means operations. In order to confer a better availability of equipment, and through them a better availability of the company, the maintenance service intervenes. It deals with problems before and after breakdowns. This improvement mainly requires a better management of the workforce and its skills. The reactivity and the organization of the maintenance service will depend on the importance of the required treatment.

It is difficult to determine precisely the required human resource number in a maintenance service (Mjema 2002). Indeed, factors making enabling capacity adaptation are prone to uncertainties. Those are due to several parameters (variations of the intervention requests which are never similar, arrival dates of requests, requests' contents, required treatment dura- tion and equipments availabilities as well as elements related to the real intervention treatments). Thus, the different tasks are well known when they occur. The reactivity and the organization of the maintenance service will depend on the importance of the required treatment.

There are mainly two types of maintenance activities: the preventive maintenance, whose activities can be long term planned, and the corrective maintenance which is related to the non foreseeable breakdowns. Within the service of maintenance, employees have different competencies and different qualification levels. Treatment speed and thus the service reactivity will depend on the choice of the employees assigned to the task.

We give in this paper a method to take care of the new tasks apparition and we propose a decision support to insert it in the current schedule. We work on the case where the task assignment has already been realized 
(for example with the heuristic presented in (Marmier et al. 2006)). The goal is to disturb as less as possible the current schedule. However, the whole schedules are subject to uncertainties and variation between theory and reality. In order to propose insertion solutions for a new task, we have to determine which places in the schedule are the more flexible in order to obtain a scheduling which would be the most robust (the less sensible to uncertainties). The fact to propose schedule solutions taking care of variation by anticipating show that our scheduling method is proactive.

In this article, we detail a methodology which will allow us to assign tasks to resources by considering disturbances. The rest of the paper is organized as followed: In the second section, we will introduce how maintenance services can be managed. In the third part, we will present the scheduling problems with uncertainties. Then we develop our model and a resolution approach. Finally, we will discuss the different obtained results.

\section{MAINTENANCE MANAGEMENT}

In scheduling and planning, the time horizon is often split in periods (the short, medium and long term). Then, we can study events on each time interval and not on a continuous scale of time. The context of this article takes place in the short term horizon. In this approach, we consider that maintenance tasks have to be scheduled when they occur (generally it is the case of corrective maintenance). The manpower is then the limiting factor in the scheduling realization. Human resources are then organized in the maintenance service which has to plan their work.

\subsection{Organization}

Within each plant, the maintenance service has to maintain equipment under operation. The level of the results to reach by the maintenance services is generally predetermined. Either a contract is signed between two (or more) partners fixed their cooperation terms, or there is a moral agreement inside the company between production and maintenance service, that fixes the equipment efficiency required. In both case, the objectives of the maintenance are defined by a level of availability (that can be different from one equipment to another). The guaranteed availability is a percentage of the opening time. If, for a machine or a group of machines, the objective of availability is not achieved, penalties have to be paid by the service provider. Conditions concerning the penalties are defined while elaborating the contract and are function of the non availability duration. We will consider in our model the minimisation of those penalties.

\subsection{Resources}

During her thesis, Agnès Letouzey carried out a study on nineteen companies to obtain their opinions on the operators' assignment problem (Letouzey 2001). It shows that operators' management, according to their competencies, is important for industry leaders and that there is still no software taking this into account. $79 \%$ of the companies think that operators' management is useful or essential in scheduling. Whereas in current softwares the operational duration is fixed, for the industry leaders, the consideration of the operators' qualification is very important to determine their assignments. For them, the qualification level has (sometimes for $47 \%$ of them and always for $27 \%$ of them) an influence over the task's duration of realization.

A maintenance service is an environment composed of $m$ operators working in parallel. We assume that all can perform each task, but not with the same efficiency. Moreover, the resource which is the most effective for a task, would not necessary be effective for all tasks. The multiplicity of competencies shows that we have a parallel machine problem, but with unrelated machines which is noted $R$ or $R_{m}|\beta| \gamma$.

\subsection{Tasks}

On a medium-term, the maintenance service has to plan and assign the best human resource for the treatment of the different maintenance tasks. Preventive and conditional maintenances have for parameters a known duration, a starting date and a completion date. The corrective maintenance tasks generally occurs in the short-term horizon. They also have a duration, which is only evaluated since it depends on a correct diagnosis. Their earliest starting date is not necessarily immediate, since spare parts are not necessarily available (they can be expected from a supplier) or the availability level of the equipment is quite good and then the intervention can be done latter.

\section{SCHEDULING PROBLEMS WITH UNCERTAINTIES}

\subsection{Problem syntheses}

In this problem while tasks have not been really treated, their data are stochastic. In order to propose a robust (and proactive) solution, our simulation will consider variations on release-dates, due-dates and of course on the duration of each tasks within the scheduling.

\subsection{Scheduling under uncertainty}

In classical scheduling problems, the data are generally supposed to be known and fixed. However, the re- 
ality does not check this hypothesis, of course because of variations, but also because a lot of data are only previsions or estimations. Optimal solutions to such scheduling problems which are based on fixed data and do not show the reality, will have only few chances to be applicable and will be subject to modifications.

In the existing model taking into account uncertainty, we find mainly the Davenport and Beck one which present three approaches: proactive, reactive and proactive-reactive approaches (Davenport and Beck 2000). Proactivity is the fact to anticipate disturbances before that they really occur. Reactive approaches work in real time, during the scheduling phases. Proactive-reactive methodologies, will try to combined both approaches in order to take into account uncertainties during all the scheduling life cycle and ensure a maximum of performance (Herroelen and Leus 2005).

A schedule is robust if this performance is few sensible to data uncertainties and variations. Moreover a schedule has to be flexible to be adaptable to the possible disturbance. We can identify a static flexibility as the temporal flexibility (concerning tasks starting date), the sequential flexibility (which authorises the permutation between tasks, and which supposes the temporal flexibility) or the assignment flexibility (which allows changing of resource after a first assignment). There is also the dynamic flexibility which is the scheduling capacity to adapt itself to disturbances (Trung 2005).

In this paper, we consider that, in a given schedule, task data are subject to more or less variations in order to be representative of the reality. Variations location will depend on the task nature. A preventive maintenance activity is well known and well documented, its face duration will be considered as determinist. However its release date depend on the current production work order end. The due date of a preventive maintenance will depend on the potential equipment breakdown due to the absence of repair. It can not be known before it occurs. Then release date and the due date, for a preventive task, can be modeled as fuzzy data. Contrary to the preventive activities, corrective maintenance tasks processing time can just be estimated. Their durations depend on a correct diagnosis. The release dates of this type of task is generally known because corrective maintenance are generally due to a breakdown and the equipment is stopped. Their due dates are also considered as known because from the breakdown, the equipment availability level goes down. Then corrective maintenance task duration can be modeled as a fuzzy data. The fact that tasks treatment required human resource implicate knowledge on their competence levels. This one being estimated (and then considered as fuzzy), the real task duration, for all type of task, will be modeled by a fuzzy duration. Finally, the most delicate disturbance, that may happen, is a new task arrival which has to be inserted in the current schedule. Its parameters are of course subject to estimation, and there precision depend on the diagnosis exactness.

\subsection{Scheduling using fuzzy logic}

Scheduling using deterministic data are useful in context where there is no source of uncertainties. However in an industrial context and especially in a maintenance environment, datas used are often estimated and have a degree of uncertainties. Solutions given by a deterministic scheduler will then not be feasible and far from the real optimum. That is why uncertainties have to consider during the modeling phase. As in many scheduling context, the main source of uncertainties is the processing time of the different tasks. The nature of each maintenance process task is fuzzy. For example the corrective maintenance tasks depend of a correct diagnosis. The notion of fuzzy is a generalization of the classical set notion where the membership of an element to a set is true or false. Fuzzy logic was introduced by Zadeh, to deal with problems where data are not deterministic (Zadeh 1965). Fuzzy set theory uses multi-valuated function to represent the membership of an object in a set rather than true or false in the classical binary theory. It quantifies how an element is considered as being in a set. Guiffrida and Nagi published a survey on fuzzy set theory applications in production (Guiffrida and Nagi 1998). A great number of work used fuzzification to represent due dates or processing time and makespan. Job earliest/latest starting date in maintenance being dependent of a fuzzy release date are of course fuzz. The tasks completion time depending of tasks predecessor are then also fuzzy. Many works had been done concerning job-shop and flow-shop problems in fuzzy environment (Petrovic et al. 2006) (Petrovic and Song 2006).

\section{MODEL}

\subsection{Tasks}

Tasks characteristics are modeled as follow : if the task $j$ is a preventive maintenance tasks:

- $p_{j}^{p}$ : face duration of the preventive maintenance task $j$.

- $\tilde{r}_{j}^{p}$ : fuzzy release date of the preventive task $j$. Uncertain release date of operation $j$ is modeled by a fuzzy set $\tilde{r}_{j}$ with a triangular membership function given by a triplet $\left(r_{j}^{1}, r_{j}^{2}, r_{j}^{3}\right)$.

- $\tilde{d}_{j}^{p}$ : fuzzy due date of the preventive task $j$. Uncertain due date of operation $j$ is modeled by a fuzzy set $\tilde{d}_{j}$ with a triangular membership function given by a triplet $\left(d_{j}^{1}, d_{j}^{2}, d_{j}^{3}\right)$.

If the task $j$ is a corrective maintenance tasks: 

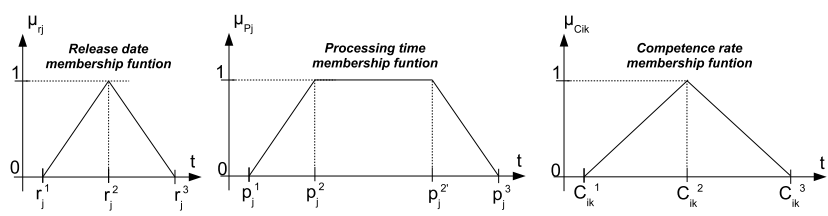

Fig. 1. Fuzzy membership functions

- $\tilde{p}_{j}^{c}$ : fuzzy face duration of the corrective maintenance task $j$. Uncertain processing time of operation $j$ is modeled by a fuzzy set $\tilde{p}_{j}$ with a 4 points shape membership function given by a quadruplet $\left(p_{j}^{1}, p_{j}^{2}, p_{j}^{2^{\prime}}, p_{j}^{3}\right)$.

- $r_{j}^{c}$ : release date of the corrective maintenance task $j$. Generally equal to the corresponding breakdown date.

- $d_{j}^{c}$ : due date of the task $j$ (this value is estimated in function of the current availability of the equipment concerned),

And for each maintenance tasks:

- $w_{j}$ : penalty which could be claimed if the treatment of the task $j$ is not performed on time.

\subsection{Human resources}

The maintenance service is composed by $m$ human resources $(i=1 \ldots m)$, characterized by a skill profile. Relative speeds do not depend only on the tasks. Each resource has a fuzzy corresponding qualification level for each task. Operators will perform them more or less quickly. The fuzzy duration of the job $j$, by the human resource $i$ is denoted by $\tilde{p}_{i j}$. With:

$$
\tilde{p}_{i j}=f\left(\tilde{p}_{j}, \tilde{C} o m p_{i k}\right), \forall i \in\{1, \ldots, m\}
$$

Where $\tilde{C} o m p_{i k}$ is the fuzzy skill rate set of resource $i$ in the competence which is required to achieve the type of task $k$. $\tilde{C} o m p_{i k}$ has a triangular membership function given by a triplet $\left(\operatorname{Comp}_{i k}^{1}, \operatorname{Comp}_{i k}^{2}, \operatorname{Comp}_{i k}^{3}\right)$. It can be represented with a matrix in which, for each different kind of job, where the corresponding rate to the required competence can be found.

$$
\left[\begin{array}{ccc}
\tilde{C} o m p_{1,1} & \cdots & \tilde{C} o m p_{1, k} \\
\vdots & \ddots & \vdots \\
\tilde{C} o m p_{m, 1} & \cdots & \tilde{C} o m p_{m, k}
\end{array}\right]
$$

Fuzzy membership function of face duration, release date and competence rate are presented on the figure 1. The treatment duration of two different tasks by two different resources enables observing that for one kind of task, a resource can be more powerful than one other, whereas, for the second task, it is the second one which is the most efficient.

In our problem, we will consider a current schedule (already computerized) which integrates $n$ tasks that had been already assigned to $m$ human resource. The current schedule can be modeled as a graph. The graph is a unit of branches which represent each one a human resource schedule. They are composed of nodes which represent tasks and arcs which are the potential constraint between to tasks (precedence). The valuations of arcs are the duration of the origin task. Tasks are placed between a fictive beginning task $B$ and fictive end task $E$. There is no link between branches, because resources work independently.

\subsection{Variables}

The variables of our problem are the following ones for each task $j$ :

- $t_{j}(j=1 \ldots n)$ : Planning date of the task $j$,

- $x_{i j}(j=1 \ldots n$ and $i=1 \ldots m): 0-1$ value representing the tasks assignment. $x_{i j}=1$ if the task $j$ is assigned to a resource $i$, else $x_{i j}=0$,

- $\tilde{C}_{i, j} \quad(j=1 \ldots n$ and $i=1 \ldots m)$ : fuzzy completion time of the task $j$, assigned to a resource $i$,

- $T_{j}(j=1 \ldots n):$ lateness of the task $j$,

- $E S_{j}(j=1 \ldots n)$ : earliest starting date of the task $j$,

- $L S_{j}(j=1 \ldots n)$ : latest starting date of the task $j$,

- $R(S)$ : Robustness measure of a schedule $S$,

\subsection{Constraints}

Each task has to be assigned only once to only one resource:

$$
\sum_{j=1}^{n} x_{i j}=1, \forall i \in\{1, \ldots, m\}
$$

A task $j$ cannot be planned before the equipment $i$ is available:

$$
\forall j, t_{j} \geqslant r_{i}
$$

\subsection{Objectives}

In order to consider corrective maintenance, we have to insert dynamically tasks in a current schedule. However it is difficult to insert tasks in a schedule which is subject to variations between the proposed one and

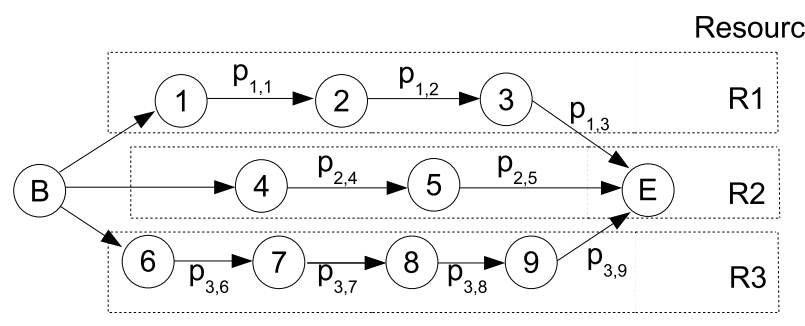

Fig. 2. Current planning 
the reality. In order to find new task insertion solutions, we have to determine which place are the most flexible and consequently propose the most robust schedule (the less sensible to variations). The fact to propose solutions taking into account variations by anticipating them, signify that our scheduling approach is proactive. Tasks which are finished late decreasing the equipment availability ratio imply that we have to minimize the total weighted tardiness.

$$
\min \sum_{j=1}^{n} w_{j} T_{j},
$$

The aim of our work being to schedule human resources activities, our methodology will take into account their individual performances to find the best resource for each task. But it will also consider the existing workload in order to distribute activities between employees.

\section{ROBUSTNESS MEASURE}

The scenario where the robustness measure intervene is a case where a new task has to inserted within a schedule. This one is add to the schedule at each place of this one and the robustness is evaluated through the measure. The schedule which will be conserved will be the one with the highest robustness measure. In case of equality the choice is given to the manager.

In order to obtain the completion time of each job, fuzzy operation have to be used. The fuzzy task duration adds to the fuzzy release date will allow to obtain the fuzzy set representing the completion time computation. As the difference with (Song and Petrovic 2005) where there is precedence constraint, here the fuzzy completion time is obtained with:

$$
\tilde{C}_{i, j}=\tilde{\max }\left(\tilde{r}_{j}, \tilde{C}_{i, \operatorname{pred}(j)}\right) \tilde{+} \tilde{p}_{i, j}
$$

where $\tilde{+}$ is the fuzzy addition operator and mãx is the fuzzy maximum operator.

Robustness measure is used to show the difference between solutions which are subject to uncertainties. It evaluates the lateness potential of a solution. A task lateness is defined by the fact that its completion date is reached after its due-date. In other words if the task is not finished at the due-date means that it will be late. In classic logic, the fact that a task $j$ is not finished, correspond to the interval $\left(-\infty ; C_{j}[\right.$. If the task is not finished before the interval $\left[D_{j} ;+\infty\right)$ the task will be late. An intersection between these intervals means that there is a lateness. In fuzzy logic the completion date and due-date will be the fuzzy intervals $\tilde{C}_{j}$ and $\tilde{D}_{j}$. Intervals previously obtained will correspond respectively to $\left(-\infty ; \tilde{C}_{j}\left[\right.\right.$ and $\left[\tilde{D}_{j} ;+\infty\right)$. Their membership functions will then be $\mu_{(-\infty ; \tilde{C}[}$ and $\mu_{\left[\tilde{D}_{j} ;+\infty\right)}$ as shown on the figure 3 (Dubois et al. 1995).
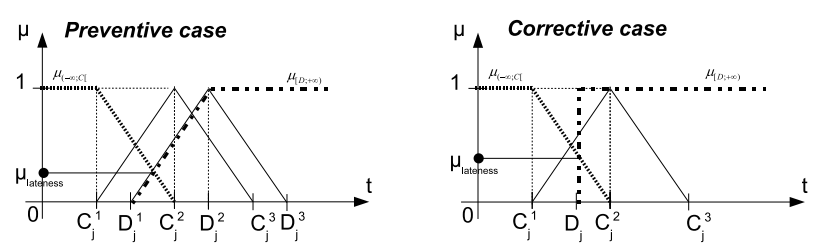

Fig. 3. Lateness possibility in case of conflict

A robust schedule is defined as being insensitive to disturbances (Leon et al. 1994). Wu and al. developed a methodology to measure scheduling robustness and a methodology to realize robust schedule in case of disruption due to control.

An other measure of the schedule robustness had been defined by Chen and Muraki (Chen and Muraki 1997) for the scheduling in batch processes. An adaptation of this measure is defined as being the average degree of conflict on the individual constraint between a task and it's due date constraint, as observed on the figure 3 . Where the fuzzy membership function $\mu_{\text {lateness }}(t)$ shows the potential lateness.

The fuzzy membership function $\mu_{\text {lateness }}(t)$ is obtain from the equation 6 . However, the robustness represents the fact that his performance is few sensible to data uncertainties and variations. The fuzzy membership function $\mu_{\text {in } \text { time }_{j}}(t)$ is then obtain from the equation 7 . Since all constraints have not the same importance we introduce the weight ${ }_{j}$ penalty factor (described in the equation 8) to weight the different conflict in the equation 9. $n$ denote all the different conflict locations within the schedule $S$ and $R(S)$ will then give its robustness level. A robust schedule will have an index $R(S)=1$ contrary to a schedule which is sensible to variation which will obtain $R(S)=0$.

$$
\begin{aligned}
& \mu_{\text {lateness }_{j}}(t)=\min \left\{\mu_{\tilde{C}_{i, j}(t)}, \mu_{\tilde{d}_{j}(t)}\right\} \\
& \mu_{\text {in }_{\text {time }}}(t)=1-\mu_{\text {lateness }_{j}}(t) \\
& \text { weight }_{j}=w_{j} / \max _{j}\left(w_{j}\right) \\
& R(S)=\frac{1}{n} \sum_{j=1}^{n} \mu_{\text {in }_{\text {time }}} * \text { weight }_{j}
\end{aligned}
$$

\section{COMPUTATIONAL RESULTS}

In order to compared and evaluated the proposed method, we will used the simulation methodology proposed in (Marmier et al. 2007). A robustness evaluation of the different insertion possibilities will be given. The different possibilities will be tried by simulation and correlation between the robustness level and the objective study will be done. 


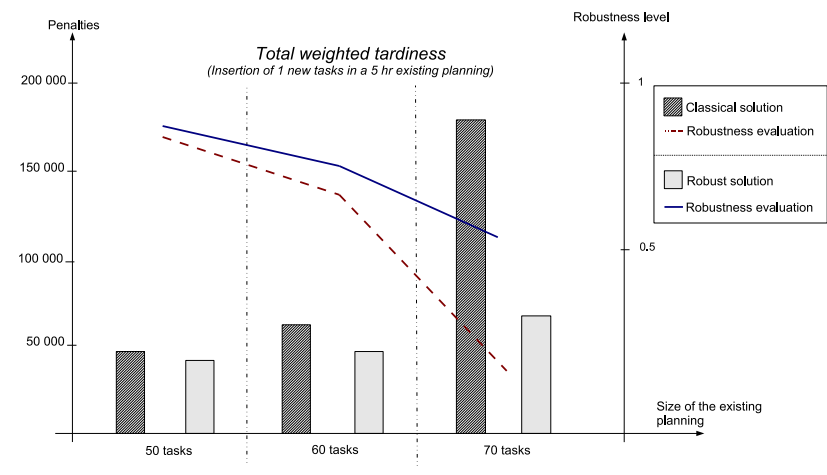

Fig. 4. Robustness evaluation

On the figure 4, we can observed the comparison between a solution which has been obtained without considering uncertainties and a robust one obtained by simulation. For both solutions we evaluated the robustness level with the methodology presented before. Tests which has been done on the figure 4 are a new task dynamic insertion in different existing planning with three different workload.

Then the observed correlation would allow us to choose the correct insertion place in an existing schedule, without simulating different cases.

\section{CONCLUSION}

In this article we developed an approach to evaluate the robustness of a schedule. It is useful when inserting a new task. We compared this approach with a simulation methodology. There is a correlation between simulation results and the robustness evaluation. This correlation signify that the robustness evaluation could give an idea from an eventual simulation result. Then both approaches can give results following the same criteria. Moreover the robustness evaluation is more quickly obtained, only few seconds comparing to simulation duration which is in minutes. A good representation of the reality is not composed of only one criteria. This correlation allow us to imagine to use the robustness evaluation with fuzzy logic as a criteria for a maintenance activities scheduling multi-criteria study.

\section{REFERENCES}

Chen, Wei and Masaaki Muraki (1997). A fuzzy evaluation of schedule robustness under processing time variations in batch plants. Journal of Chemical Engineering of Japan 30(2), 260-267.

Davenport, Andrew J. and J. Christopher Beck (2000). A survey of techniques for scheduling with uncertainty. Technical report. IBM and Ilog.

Dubois, Didier, Hélène Fargier and Henri Prade (1995). Fuzzy constraints in job-shop scheduling. Journal of Intelligent Manufacturing 6(4), 215234.
Guiffrida, Alfred L. and Rakesh Nagi (1998). Fuzzy set theory applications in production management research: a literature survey. Journal of Intelligent Manufacturing 9, 39-56.

Herroelen, Willy and Roel Leus (2005). Project scheduling under uncertainty : Survey and research potentials. European Journal of Operational Research 165, 289-306.

Leon, V. Jorge, S. David Wu and Robert H. Storer (1994). Robustness measures and robust scheduling for job shops. IIE Transactions.

Letouzey, Agnès (2001). Ordonnancement interactif basé sur des indicateurs : Application à la gestion de commandes incertaines et à l'affectation des opérateurs. PhD thesis. Institut National Polytechnique de Toulouse.

Marmier, François, Christophe Varnier and Noureddine Zerhouni (2006). Maintenance activities scheduling under competencies constraints. In: ICSSSM.

Marmier, François, Christophe Varnier and Noureddine Zerhouni (2007). Dynamic scheduling of maintenance activities under uncertainties. In: International Conference on Industrial Engineering and Systems Management (IESM).

Mjema, E.A.M. (2002). An analysis of personnel capacity requirement in the maintenance departement by using a simulation method. Journal of Quality in Maintenance Engineering 8(3), 253273.

Petrovic, Dobrila, Alejandra Duenas and Sanja Petrovic (2006). Decision support tool for multiobjective job shop scheduling problems with linguistically quantified decision functions. Decision Support Systems.

Petrovic, Sanja and Xueyan Song (2006). A new approach to two-machine flow shop problem with uncertain processing times. Optimization and Engineering 7, 329-342.

Song, Xueyan and Sanja Petrovic (2005). Handling fuzzy constraints in flow shop problem. In: $E U$ ROFUSE.

Trung, La Hoang (2005). Utilisation d'ordres partiels pour la caractérisation de solutions robustes en ordonnancement. $\mathrm{PhD}$ thesis. Laboratoire d'Analyse et d'Architecture des Systèmes du CNRS.

Zadeh, L. A. (1965). Fuzzy sets. Information and control 8, 1265-1279. 